\title{
Decompression, Enucleation and Carnoy's Solution as a Conservative Management of an Odontogenic Keratocyst: A Case Report
}

\author{
Andres Pauwels ${ }^{a, *}$, Costanza E. Lozano ${ }^{b}$, Juan Pablo López , Juan C. Munevard \& Sergio M. Viafara-Garcia
}

\section{SUMMARY}

Odontogenic keratocyst (OKC) is a controversial pathology in terms of designation and treatment because it is a locally aggressive cyst that through its history has had different names and a number of alternatives for its management, such as enucleation, cryotherapy, decompression, Carnoy's solution (CS) application or peripheral osteotomy. All the techniques having different results in relapse rates some with more advantages than others; however, until now there are no studies with sufficient data to define which is the best surgical technique to treat the OKC. We report the case of a 48-year-old man diagnosed with OKC who was treated combining different techniques. Further follow-up at the 3-, 6-, 10- and 14-month marks and three years, showed complete recovery and no evidence of recurrence. Therefore large and invasive mandibular cysts could be treated by decompression, followed by enucleation, peripheral ostectomy and then the application of CS to the bony cavity. This approach seems to be effective in the management of large and invasive mandibular cysts $\mathrm{OKC}$ attenuating recurrence until long follow up and impacting quality life instead of invasive techniques such as hemimandibulectomy.

\footnotetext{
a Oral and Maxillofacial Surgeon, Professor Universidad El Bosque. Department of Oral Health, Hospital Universitario Fundación Santa Fe de Bogotá, Bogotá, D.C., Colombia.

${ }^{b}$ Oral and Maxillofacial Surgeon, Professor Universidad El Bosque. Department of Oral Health, Hospital Universitario Fundación Santa Fe de Bogotá, Bogotá, D.C., Colombia.

c Oral and Maxillofacial Research, Fundación Santa

Fe de Bogotá, Bogotá Colombia.

Private practice, Bogotá, D.C., Colombia.

${ }^{d}$ Associate Professor, Unit of Oral Basic investigation (UIBO), School of Dentistry, Universidad El Bosque, Bogotá, D.C., Colombia. Department of Oral Health, Hospital Universitario Fundación Santa Fe de Bogotá, Bogotá, D.C., Colombia.

${ }^{\text {e }}$ Assistant Professor, Unit of Oral Basic investigation (UIBO), School of Dentistry, Universidad El Bosque, Bogotá, D.C., Colombia. Department of Oral Health, Hospital Universitario Fundación Santa Fe de Bogotá, Bogotá, D.C., Colombia.

Centro de Investigación e Innovación Biomédica (CIIB), Universidad de Los Andes, Santiago, Chile.
}

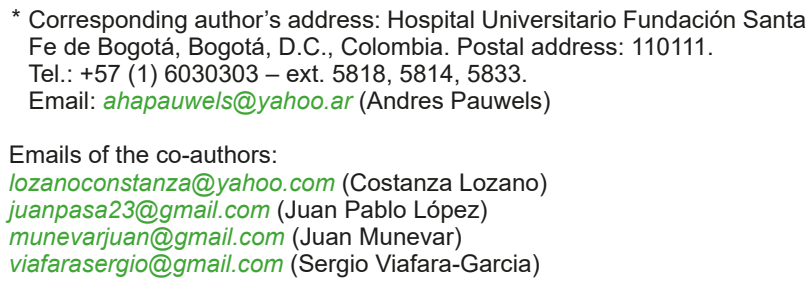

* Corresponding author's address: Hospital Universitario Fundación Santa Fe de Bogotá, Bogotá, D.C., Colombia. Postal address: 110111. Tel.: +57 (1) 6030303 - ext. 5818, 5814, 5833.

Email: ahapauwels@yahoo.ar (Andres Pauwels)

Emails of the co-authors:

lozanoconstanza@yahoo.com (Costanza Lozano)

juanpasa23@gmail.com (Juan Pablo López)

munevarjuan@gmail.com (Juan Munevar)

viafarasergio@gmail.com (Sergio Viafara-Garcia)

Please cite article as: Pauwels A, Lozano CE López JP, Munevar JC, Viafara-Garcia S M. Decompression, enucleation and Carnoy's solution as a conservative management of an odontogenic keratocyst: a case report. $J$ Diagn Treat Oral Maxillofac Pathol 2020;4(7):119-24.

Paper received 27 March 2020

Accepted 6 May 2020

Available online 31 July 2020

httpi://dx.doi.org/10.23999/j.dtomp.2020.7.3.

(C) 2020 OMF Publishing, LLC. This is an open access article under the CC BY license (http://creativecommons.org/licenses/by-nc/4.0/). 


\section{INTRODUCTION}

$\mathrm{O}$ dontogenic keratocyst (OKC) is an odontogenic cyst whose name has presented multiple changes in the last years due to its aggressive behavior and its high rate of recurrence in the surgical site. ${ }^{1}$ It was first described in 1876 and classified by Phillipsen in 1956 and although in 2005 the World Health Organization (WHO) recommended the term cystic neoplasm, ${ }^{1}$ currently, the OKC returned its original nomenclature. ${ }^{2,3}$ In the maxilla, OKC has been considered as the main odontogenic and aggressive cysts with an important recurrence rate in the mandibular angles. ${ }^{1}$ It can be a unilocular or multilocular lesion with sclerotic borders and cortical expansion frequently associated with missing tooth. ${ }^{1}$ Histologically, the parakeratinized epithelium is lined of five to eight cell layers with areas of squamous metaplasia ${ }^{4,5}$ and it has been described as a primary cysts of the jaws, related with skeletal abnormalities as the bifid ribs, hand and foot abnormalities, beside of alterations in the skin associated to basal cell carcinomas.

The OKC treatment is also controversial despite of the enucleation is used as basic management in detaching the cyst from the bone cavity, complementary techniques have arisen. Some authors reported that the treatment with enucleation alone can result in a $60 \%$ of recurrences. ${ }^{6}$ Thus, other adjuvant treatments have been used. For instance, the previous decompression minimizes tissue damage, and is one of the most common therapeutic options mainly used in larges cases of OKC. However its effectiveness is questioned due to the high recurrence of the OKC which has increased the interest to combine with others adjuvants techniques such as the application of Carnoy's solution (CS) or marginal ostectomy with rotary instruments, both frequently used after enucleation as a complement which seeks to eliminate adjacent satellite cyst bone tissue. ${ }^{7,8}$ Nevertheless, other authors reported that even with CS or peripheral osteotomy the recurrences varied from 0 to $100 \%$. $^{9}$ Thus, although there is not a clear reduction in the recurrences yet, the treatment of OKC using CS with marginal ostectomy as adjuvant treatment have been proposed to surgically husking the cyst out of the bone without leaving any macroscopic remnant mainly through the cauterizing effect of CS. ${ }^{10}$

Our aim in this OKC clinical case report is addressing its clinical, radiographic, and treatment aspects, focusing on contributing to a better understanding of the complementary techniques as decompression and CS to minimize damage tissue and recurrence rate through a conservative protocol.

\section{CASE}

This case report conformed to precepts of the Declaration of Helsinki on medical protocol and ethics and was approved by the Hospital Ethics Committee. Informed consents were signed by the participant and exemption was granted given the use of de-identified patient variables.

A 48-year-old man was referred to the Oral and Maxillofacial Surgery Service at Hospital Universitario Fundación Santa Fe de Bogotá (Bogotá, D.C., Colombia), with a chief complaint of intermittent discomfort and slight pain on the right side near the mandibular angle and slowly progressing swelling of 7 months duration.

At the initial consultation, the patient reported a history of hypertension that was well controlled. Clinical extraoral examination showed mild intraoral contour deformity and the mandibular nerve was intact with no compromise in sensation and there is not compromise of facial soft tissue. Intraoral examination revealed a well-defined, localized swelling, fluctuant, and non-tender on palpation. The overlying mucosa was the same color as that of adjacent mucosa and presented with no sign of inflammation.

The panoramic radiograph correlated with the clinical findings showing a well-defined multilobular radiolucent lesion, cystic-appearing lesion with a thin sclerotic rim which encompassed most of the right mandibular ramus that extended from the 47 until mandibular notch and the lesion measured $4.2 \times 3.3 \mathrm{~cm}$ (Fig 1).

A fine-needle aspiration revealed a yellowcolored highly viscous fluid, then incisional biopsy of the cyst was performed in addition to placement of a decompression tube (Fig 2). The histopathological analysis and features with hematoxylin and eosin stain showed an OKC pattern lesion. 

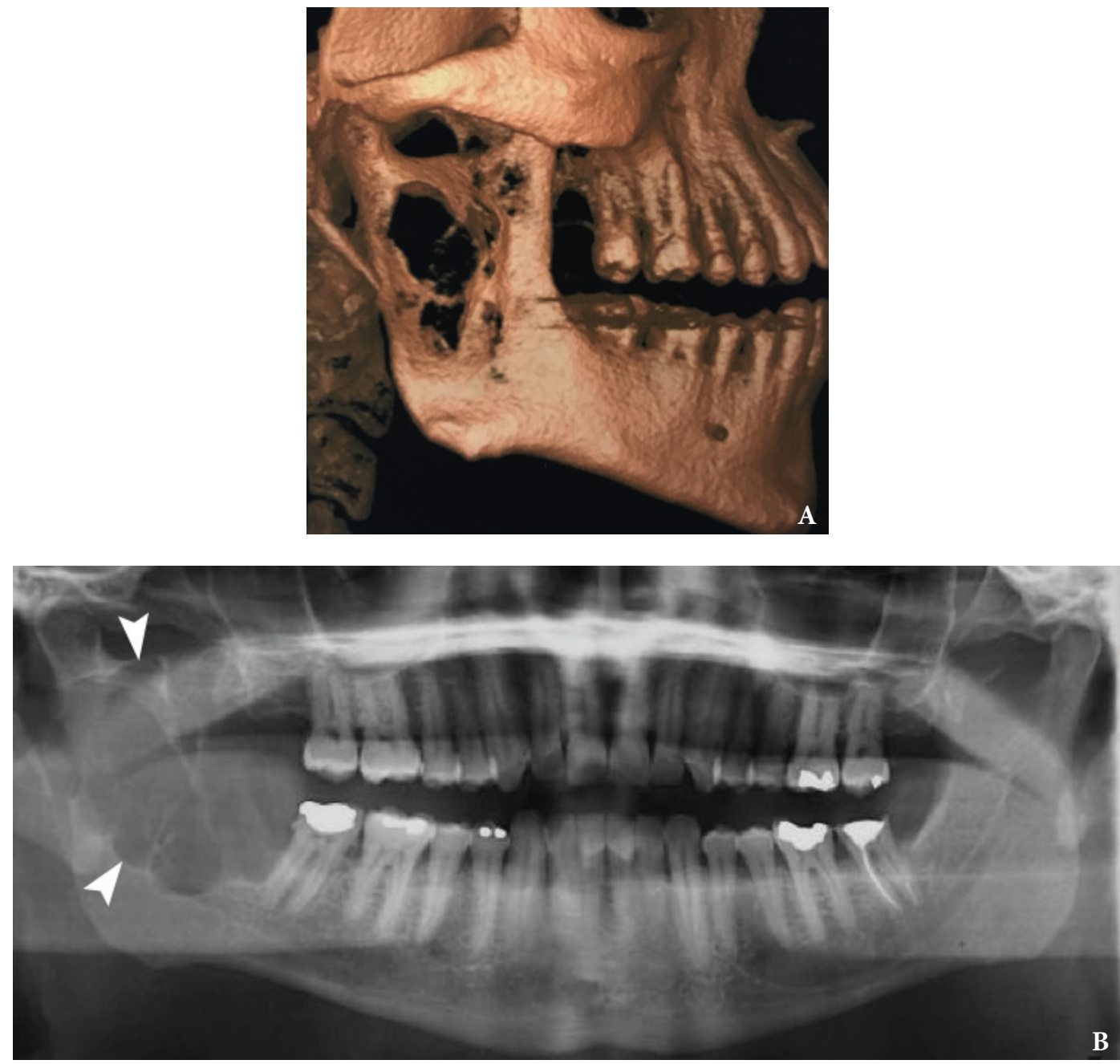

FIGURE 1. Three-dimensional computed tomography reconstruction $(\mathbf{A})$ of the right mandibular ramus with OKC in a 48-year-old man. Panoramic radiography $(\mathbf{B})$ shows that OKC (arrowheads) was measured $4.2 \times 3.3 \mathrm{~cm}$ and extended from the tooth 47 to mandibular notch. Both images correlated with the clinical findings showing a well-defined multilobular radiolucent lesion.

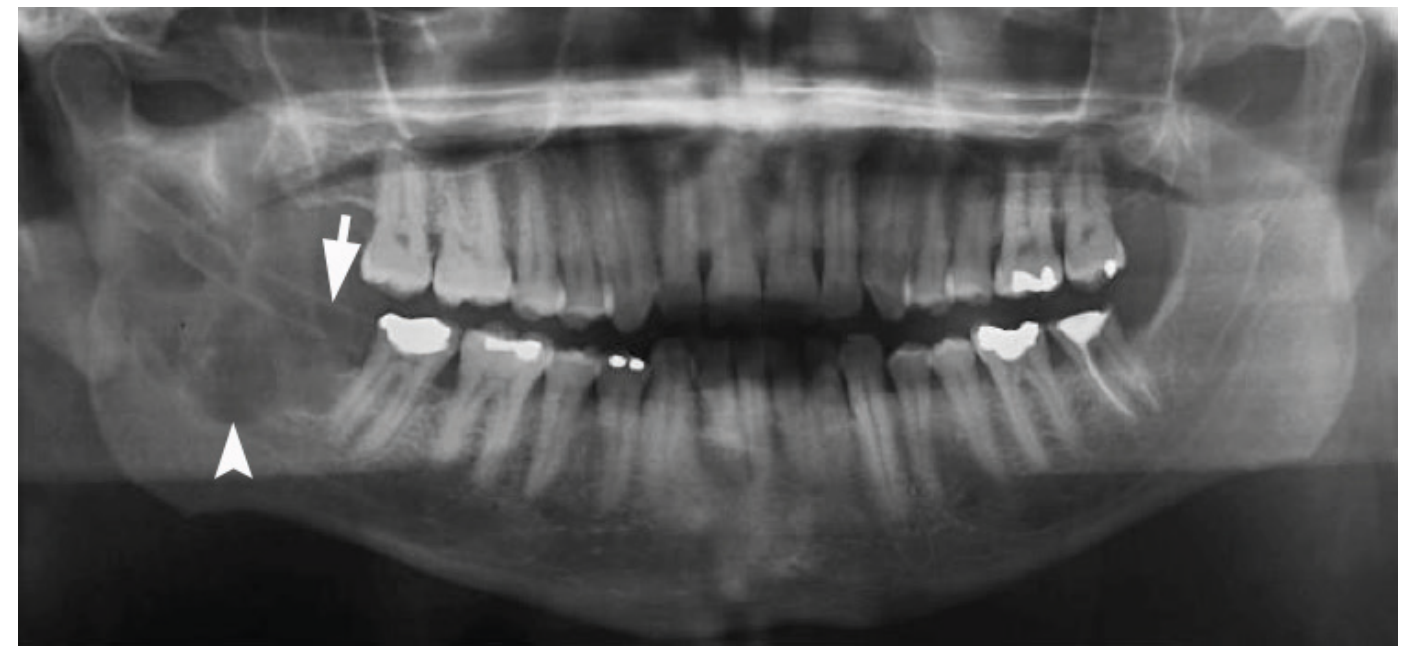

FIGURE 2. A decompression tube (arrow) on large multilocular lesion (arrowhead) with variable sclerotic margins in the right mandibular ramus. 
Owing to the close location of the lesion to mandibular nerve the patient was compliant with three-times-daily direct flushes of saline rinses through the decompression tube in addition to monthly routine clinical follow-ups.

After 10 months, a panoramic imaging showed a considerable 3-dimensional regression of the lesion, which showed a bony rim surrounding the decompressed lesion of $1 \times 1 \mathrm{~cm}$ (Fig 3 ).

Once it noted the successful outcome of decompression, the patient was scheduled for the third stage of the planned surgical procedure of enucleation. A sulcular incision was made with a posterior extension to create a triangular flap. Then, the lesion was exposed by creating a bony window using piezoelectric. The lesion was enucleated, including the fistulous tract previously created by the decompression tube. After enucleation, curettage was completed not only by removing a layer of the surrounding bone using a round bur, but also with the use of CS both strategies for preventing recurrence of satellite cysts. The wound was closed primarily. The cyst specimen was sent for histopathological evaluation where the final diagnosis was confirmed as an OKC. The patient returned 1 week later to assess post-surgical wound healing, and returned for further follow-up at the 3-, 6-, 10- and 14-month marks and three years, which showed complete recovery and no evidence of recurrence (Fig 4).

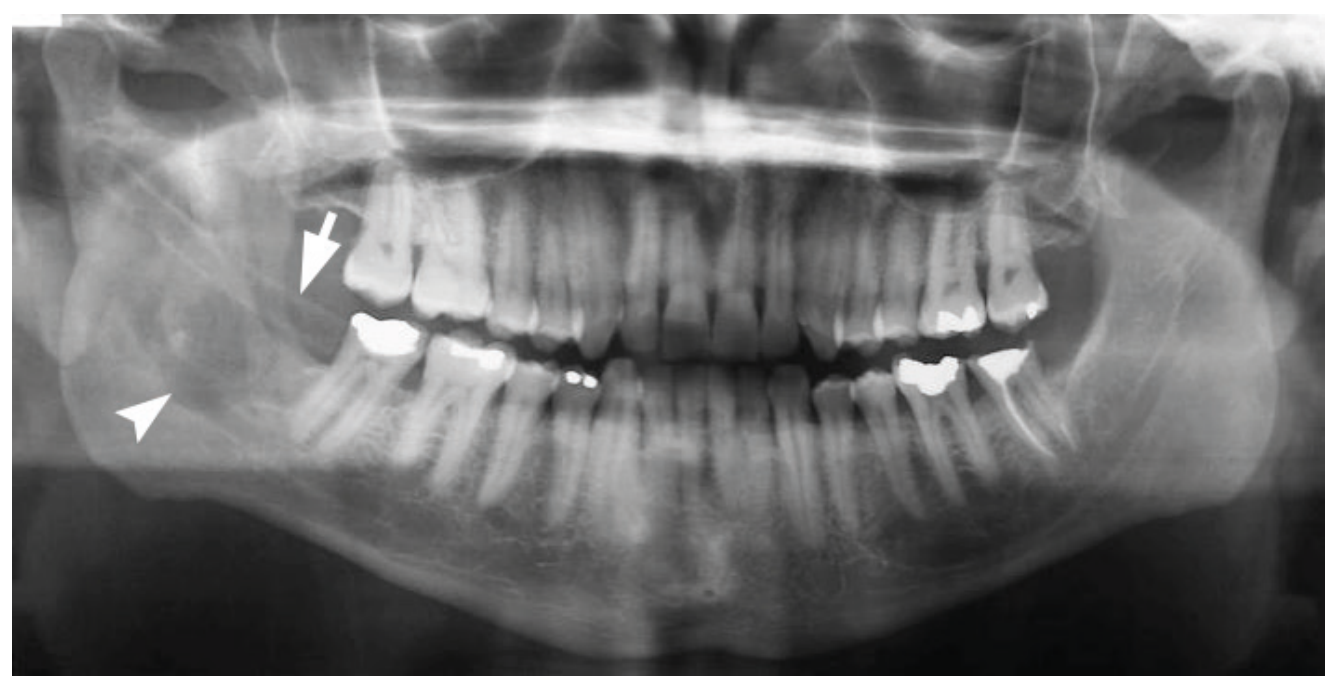

FIGURE 3. 10-month of the decompression treatment performed in order to reduce the cyst (arrowhead) with a decompression tube (arrow) sutured into the ramus.

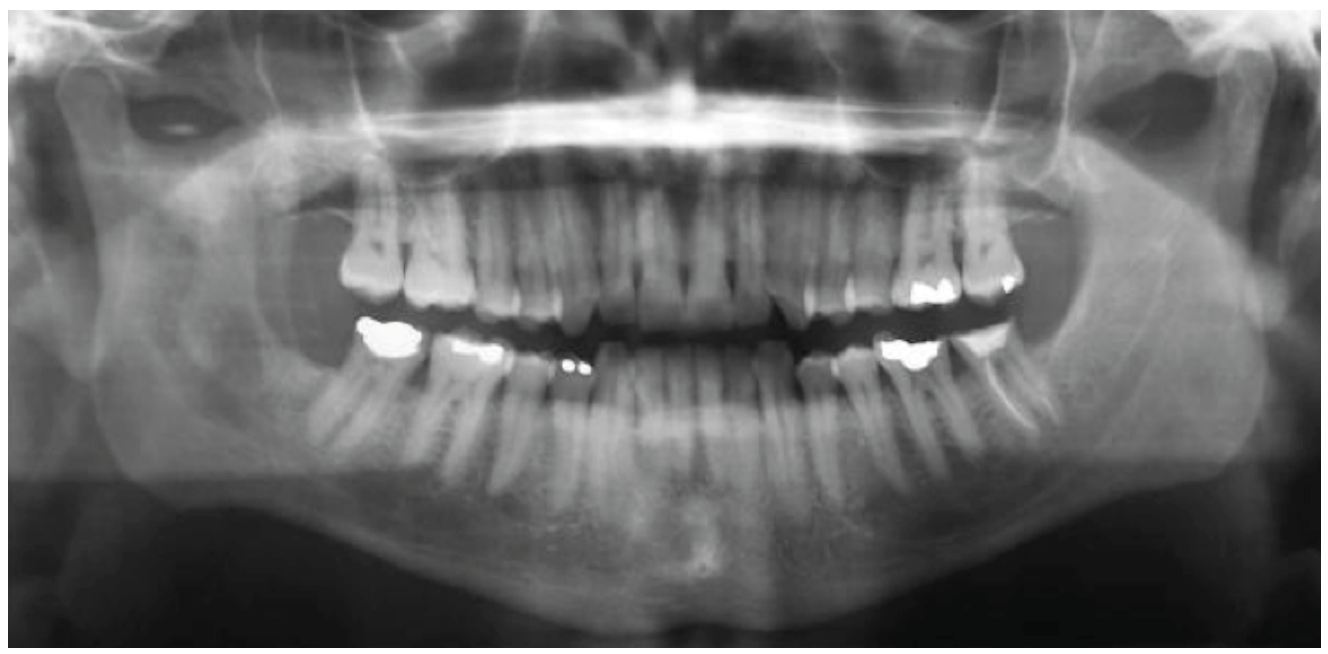

FIGURE 4. Bone repair 3 years after enucleation. 


\section{DISCUSSION}

Odontogenic cysts are relatively common lesions, however, it is the one of the rare odontogenic cysts, which attracts many researchers, surgeons and pathologists due to its unique characteristics as a highly aggressive and destructive behavior.

The controversial classification of odontogenic cysts also have been transferred toward the surgical treatment, which is mainly categorized in conservative or aggressive surgical approach. ${ }^{11}$ The conservative treatment is "cyst oriented" and thus includes decompression, enucleation, with or without curettage or marsupialization; while aggressive treatment is considering in OKC with "neoplastic nature" or friable properti which contribute to its high recurrence rate, and some adjuvant treatments such as a marginal ostectomy and chemical curettage with CS have been proposed. ${ }^{7,8,11,12}$

Large lesions, recurrent cases, and syndromic patients are mostly treated with aggressive approach, but paradoxically, there have been increasing reports that $\mathrm{OKC}$ can be treated for partial cure, or even complete cure. ${ }^{13,14}$ Minor total recurrence rates has been observed after decompression followed by enucleation (11.9\%), which suggest a significant superiority of success for OKC treatments that use decompression followed by enucleation, instead of an initial enucleation or decompression alone. ${ }^{13}$ Thus, the histological changes by the decompression generated in the cystic capsule decrease the intramural pressure, that allows the healthy bone to gain space or the gradual reduction of the cyst. ${ }^{15}$ However, according to meta-analysis, this histological changes seems not be enough in large lesion with typical neoplastic behavior or persistent growth after incomplete removal. ${ }^{14}$

Some authors have reported that the size of the lesion affects the recurrence rate, suggesting a conservative approach for small OKC (maximum $1 \mathrm{~cm}$ in diameter) near alveolar process, and aggressive management for larger lesions near the base of the skull that has invaded soft tissue. ${ }^{16}$ Likewise, different authors have demonstrated that in large lesions the effectiveness of conservative treatment increases in combination with adjunctive measures, which is associated with minor recurrence rates. ${ }^{17}$ The use of $\geq 2$ adjuvant treatments have reduced the recurrence range between 0 and 7.9 percent. ${ }^{17}$ Therefore the decompression followed by enucleation can be combined with CS. ${ }^{17,18}$ For this reason the purpose of this case report was to highlight the importance of conservative treatment with adjuvant techniques that minimize the damaged tissue to preserve anatomical structures and reduce morbidity to the patient which is mostly recommended for large lesions, recurrent cases and syndromic patients with "neoplastic nature" of OKC. . $^{11,12}$

The CS is prepared with ethanol, chloroform, glacial acetic acid and ferric chloride and applied in the cystic cavity during three minutes, followed by a profuse saline rinse wash. This technique has important results in the elimination of recurrent satellite cysts in combination with enucleation and decompression to prevent relapse. ${ }^{2}$ The CS has even shown a recurrence of $0 \%$ with a follow-up of three years, ${ }^{18}$ while modified CS without chloroform showed a rate of $35 \%$, almost 7 times more likely to recurrence, which suggests the use of chloroform in the CS. ${ }^{19}$

\section{CONCLUSIONS}

Therefore, despite of absence quality evidence with specific cohort interval that supports the OKC management with chloroform-CS or without chloroform, we recommend that large and invasive mandibular cysts could be treated in combination by decompression, followed by enucleation, peripheral ostectomy and then the application of CS to the bony cavity for no more than 3 minutes which appears to be efficient for treating of OKC by diminishing the recurrence during the most critical period (the first 3 years).

\section{ACKNOWLEDGMENTS}

This study was supported in part funding from the Oral Health Service, Hospital Universitario Fundación Santa Fe de Bogotá, Colombia.

\section{DECLARATION OF INTEREST}

This research was funded through Hospital Universitario Fundación Santa Fe de Bogotá, Colombia; the authors have expressed none conflict of interests. 


\section{ROLE OF CO-AUTHORS}

Pauwels A. (material processing).

Lozano C.E. (material processing).

Lopez J.P. (writing and editing)

Munevar J.C. (concept and design).

Viafara-Garcia S.M. (writing and editing).

\section{REFERENCES}

1. Pogrel MA. The keratocystic odontogenic tumor. Oral Maxillofac Surg Clin North Am 2013;25(1):21-30.

2. Ribeiro-Júnior O, Borba AM, Alves CAF, GouveiaMMd, Deboni MCZ, Naclério-Homem MdG. Reclassification and treatment of odontogenic keratocysts: a cohort study. Braz Oral Res 2017;18;31:e98.

3. Kennedy RA. WHO is in and WHO is out of the mouth, salivary glands, and jaws sections of the 4th edition of the WHO classification of head and neck tumours. Br J Oral Maxillofac Surg 2018;56(2):90-5.

4. Han Y, Fan X, Su L, Wang Z. Diffusion-weighted MR imaging of unicystic odontogenic tumors for differentiation of unicystic ameloblastomas from keratocystic odontogenic tumors. Korean J Radiol 2018;19(1):79-84.

5. Pogrel MA. The history of the odontogenic keratocyst. Oral Maxillofac Surg Clin North Am 2003;15(3):311-5.

6. Sharif FN, Oliver R, Sweet C, Sharif MO. Interventions for the treatment of keratocystic odontogenic tumours (KCOT, odontogenic keratocysts (OKC)). Cochrane Database Syst Rev 2010;(9):CD008464.

7. Güler N, Sençift K, Demirkol O. Conservative management of keratocystic odontogenic tumors of jaws. ScientificWorldJournal 2012;2012:680397.

8. Johnson NR, Batstone MD, Savage NW. Management and recurrence of keratocystic odontogenic tumor: a systematic review. Oral Surg Oral Med Oral Pathol Oral Radiol 2013;116(4):e271-6.

9. Ecker J, Horst RT, Koslovsky D. Current role of Carnoy's solution in treating keratocystic odontogenic tumors. J Oral Maxillofac Surg 2016;74(2):278-82.

10. Ribeiro JO, Borba AM, Alves CaF, de Gouveia MM, Coracin FL, Guimarães Júnior J. Keratocystic odontogenic tumors and Carnoy's solution: results and complications assessment. Oral Dis 2012;18(6):548-57.

11. Morgan TA, Burton CC, Qian F. A retrospective review of treatment of the odontogenic keratocyst. J Oral Maxillofac Surg 2005;63(5):635-9.

12. Kaczmarzyk T, Mojsa I, Stypulkowska J. A systematic review of the recurrence rate for keratocystic odontogenic tumour in relation to treatment modalities. Int J Oral Maxillofac Surg 2012;41(6):756-67.

13. de Castro MS, Caixeta CA, de Carli ML, Ribeiro Júnior NV, Miyazawa M, Pereira AAC, Sperandio FF, Hanemann J. Conservative surgical treatments for nonsyndromic odontogenic keratocysts: a systematic review and meta-analysis. Clin Oral Investig 2018;22(5):2089-101.

14. Li T. The odontogenic keratocyst: a cyst, or a cystic neoplasm? J Dent Res 2011;90(2):133-42.

15. Oliveros-Lopez L, Fernandez-Olavarria A, TorresLagares D, Serrera-Figallo MA, Castillo-Oyagüe R, Segura-Egea JJ, Gutierrez-Perez JL. Reduction rate by decompression as a treatment of odontogenic cysts. Med Oral Patol Oral Cir Bucal 2017;22(5):e643-50.

16. Dammer R, Niederdellmann H, Dammer P, NueblerMoritz M. Conservative or radical treatment of keratocysts: a retrospective review. $\mathrm{Br} J$ Oral Maxillofac Surg 1997;35(1):46-8.

17. Pogrel MA. Keratocystic odontogenic tumors. J Oral Maxillofac Surg 2016;74(3):438-9.

18. Alchalabi NJ, Merza AM, Issa SA. Using Carnoy's solution in treatment of keratocystic odontogenic tumor. Ann Maxillofac Surg 2017;7(1):51-6.

19. Dashow JE, McHugh JB, Braun TM, Edwards SP, Helman JI, Ward BB. Significantly decreased recurrence rates in keratocystic odontogenic tumor with simple enucleation and curettage using Carnoy's versus modified Carnoy's solution. J Oral Maxillofac Surg 2015;73(11):2132-5. 